\title{
Avaliação do sistema de vigilância entomológica da doença de Chagas com participação comunitária em Mambaí e Buritinópolis, Estado de Goiás
}

\author{
Assessment of an entomological surveillance system for Chagas disease \\ with community participation in Mambaí and Buritinópolis, State of Goiás
}

\author{
Antonio Carlos Silveira ${ }^{1}$, Dilermando Fazito de Rezende ${ }^{2}$, Ana Maria Nogales ${ }^{3}$, \\ Juan José Cortez-Escalante ${ }^{1}$, Cleudson Castro ${ }^{1}$ e Vanize Macêdo ${ }^{1 \dagger}$
}

\begin{abstract}
RESUMO
A vigilância entomológica da doença de Chagas em Mambaí e Buritinópolis, no Estado de Goiás, Brasil, tem sido mantida com participação da população, notificando a presença de vetores nas habitações. Passado longo tempo após instituídas as ações de controle e tendo-se já certificado a interrupção da transmissão vetorial, buscou-se avaliar o conhecimento e as práticas da população nessa situação. Os resultados apontam progressivo desinteresse pelo tema doença de Chagas, atribuível à redução da magnitude do problema representado pela enfermidade, a pouca participação das escolas na vigilância, à pequena importância dos vetores secundários e nativos e, em consequiência, às limitadas intervenções dos serviços de controle em resposta às notificações. Propõe-se, que atividades de busca direta por amostragem sejam periodicamente realizadas e maior envolvimento das instituições de ensino.
\end{abstract}

Palavras-chaves: Doença de Chagas. Vigilância entomológica. Participação comunitária.

\begin{abstract}
Entomological surveillance of Chagas disease in Mambaí and Buritinópolis, in the State of Goiás, Brazil, has been kept up through the local population's participation, consisting of reporting the presence of vectors inside their homes. A long time has elapsed since instituting these control measures and it has now been certified that vector transmission has been halted. Thus, this study sought to evaluate the population's knowledge and practices in this situation. The results show that there has been progressive indifference towards the topic of "Chagas disease", which can be attributed to the reduction in the magnitude of the problem that this disease represented, little participation in surveillance among schools, low epidemiological importance of secondary and native vectors and, consequently, limited control interventions from health services in response to notifications. It is proposed that direct search activities by means of sampling should be carried out periodically, and that there should be greater involvement among teaching institutions.
\end{abstract}

Key-words: Chagas disease. Entomological surveillance. Community participation.

O controle da transmissão vetorial da doença de Chagas no Brasil foi estruturado na forma de programa de alcance nacional a partir de 1975. Antes, foram cumpridas ações isoladas, de maior ou menor alcance, em função de um aporte descontínuo de recursos, determinado pelo baixo nível de prioridade conferido às atividades. Com o controle da malária nas regiões Sudeste, Nordeste e Centro-Oeste do país, e os recursos que resultaram excedentes, passou-se a contar com os meios necessários para intervenções abrangentes e mantidas em caráter regular.

\footnotetext{
1. Núcleo de Medicina Tropical, Universidade de Brasília, Brasília, DF. 2. Faculdade de Medicina de Barbacena, Barbacena, MG. 3. Departamento de Estatística da Universidade de Brasília, DF. $1 \dagger$ In memorian

Endereço para correspondência: Dr. Cleudson Castro. Núcleo de Medicina Tropical/UnB. Caixa Postal 4517, 70904-970 Brasília DF.

Fax: $55613273-2811$

e-mail: tropical@unb.br

Recebido para publicação em 31/01/2008

Aceito em 03/02/2009
}

Desde o final da década de 40 se dispunha de tecnologia eficaz para o controle de populações domiciliadas de triatomineos ${ }^{2415}$, mas as prioridades entre as doenças transmitidas por vetores (DTVs) eram outras: doenças agudas com transmissão epidêmica e risco de ocorrência no meio urbano, eram, além da malária, a peste e a febre amarela.

0 controle da doença de Chagas não apenas herdou recursos da malária, mas o modelo de operação campanhista de outras enfermidades. Seguia em parte a metodologia de trabalho definida a partir do pressuposto de que as chamadas grandes endemias, pela sua magnitude e intensidade da transmissão, deveriam de início receber ataque intensivo e de larga abrangência. Essas ações seriam sustentadas por algum tempo, até que alcançadas determinadas metas, previamente estabelecidas e definidas como condição para a instituição do que se convencionou chamar de fase de vigilância. Assim, a vigilância corresponderia a uma fase avançada das operações, para a qual se admitia que os serviços locais de saúde, poderiam assumir as tarefas próprias de controle das DTVs. 
No caso da doença de Chagas, um componente essencial da vigilância epidemiológica, em função da pouca aparência clínica do caso agudo, é aquela sobre o vetor, a ser exercida de forma contínua e duradoura, e para isso, a participação da população é indispensável. Não apenas para a sustentabilidade das ações, mas porque é o método mais sensível de pesquisa entomológica para triatomíneos, em situações em que as densidades das populações domiciliadas são mínimas ${ }^{11217}$.

A área eleita, tem uma longa história de trabalho no controle da doença de Chagas. Desde 1974 o Núcleo de Medicina Tropical da Universidade de Brasília (UnB) vem atuando no município de Mambaí. Os levantamentos de base foram realizados em 1975 através de inquérito entomológico que abarcou todas as unidades domiciliares (UDs) e estudos clínicos, eletrocardiográficos, radiológicos e de soroprevalencia em mais de 3.000 habitantes $^{367}$. Em 1979, novo inquérito parcial (455 UDs de um total de 876 então existentes) revelou um aumento da infestação de 52 para $74 \%{ }^{10}$.

Em 1980, a Superintendência de Campanhas de Saúde Pública fez o tratamento integral, com inseticida clorado (BHC), de todos os domicílios, seguido de rociamento seletivo por UD infestada no ano seguinte ${ }^{9}$. A partir de 1983 foram empregados inseticidas piretroides, aplicados seletivamente em unidades com infestação residual ou reinfestadas, conhecidas através de vigilância entomológica exercida por busca ativa do vetor ou com a informação gerada por unidades mínimas de vigilância $(\mathrm{UMV})^{8}$.

As unidades mínimas de vigilância e outros métodos de controle foram utilizados a partir do reconhecimento de que a sustentabilidade das ações de vigilância entomológica dependeria da participação da populaçãa $0^{5}$. A princípio se fez a distribuição de sacolas plásticas para a coleta de triatomineos pelos moradores, após o tratamento das habitações com inseticida. A resposta não correspondeu ao que se esperava, o que levou à iniciativa de se realizar inquérito sobre o conhecimento, atitudes e práticas da população com respeito ao vetor e à doença de Chagas ${ }^{1}$.

Passados aproximadamente 25 anos, desde aquele estudo, se buscou novamente avaliar o conhecimento da população hoje residente na área, quando já interrompida a transmissão vetorial, e virtualmente eliminado o Triatoma infestans e com a presença episódica de Triatoma sordida nos domicílios, vetor pouco competente na transmissão domiciliar da doença ${ }^{18}$. Importante ressaltar que as atividades de vigilância e controle continuam até hoje tanto por parte do município com pela Universidade de Brasília.

0 presente trabalho é também complementar a dois outros ${ }^{13} 18$ que, em conjunto, se poderia qualificar como uma avaliação das ações de controle e vigilância da endemia chagásica nesses municípios, desenvolvidas pelo NMT/UnB em associação com o Ministério da Saúde.

\section{MATERIAL E MÉTODOS}

Trata-se de um estudo de corte transversal, sobre aspectos diversos da transmissão da doença de Chagas realizado em julho de 2004, através do levantamento do nível de conhecimento de residentes dos municípios de Mambaí e Buritinópolis, em
Goiás. 0 estudo consistiu da aplicação de questionário sobre um conjunto de características da doença e seu agente transmissor, em amostra da população urbana e rural dos municípios de Mambaí e Buritinópolis, visando determinar o grau de conhecimento a respeito dos determinantes fundamentais da transmissão da endemia.

Para a seleção dos participantes do estudo, considerou-se uma amostra de 407 residências que representa 13,8\% das UDs das áreas urbana e rural, de diferentes localidades de ambos os municípios, selecionados por amostragem aleatória e cujos habitantes foram considerados potencialmente aptos para participar. Foram incluídos no estudo os ocupantes dos domicílios habilitados a responder às questões e que estavam na residência no momento da visita dos agentes do inquérito. Foram, dessa forma, selecionados 767 indivíduos de ambos os sexos, com sete anos de idade ou mais, para compor a amostra.

o questionário aplicado foi elaborado especificamente para a ocasião e era composto de questões relativas a aspectos particulares do inseto vetor e da doença; ao local de nascimento e residências atuais e anteriores e características pessoais, além da identificação dos domicílios e seus ocupantes.

Os questionários foram aplicados aos participantes, nos domicílios das pessoas, por médicos do Curso de Pós-Graduação em Medicina Tropical, durante estágio realizado na área. Cada domicílio foi visitado uma única vez. Os questionários foram preenchidos durante as entrevistas, com as respostas dadas pelo entrevistado às questões, em nenhum momento foi preenchido pelo próprio entrevistado. As variáveis do estudo correspondem, integralmente, às questões do questionário acima descrito. A análise dos dados foi realizada em computadores tipo PC, com recursos de processamento estatístico do software EPIINFO, versão 3.3.2 e de programas de uso comercial, após transcrição dos registros para meio magnético, por digitação.

Foram feitas as distribuições de frequiências e calculadas as médias, desvios padrões e percentuais indicados para cada tipo de dado. No cálculo dos intervalos de confiança considerou-se como delineamento amostral a amostragem aleatória simples. 0 nível de significância adotado na análise foi de $5 \%$ e o projeto de pesquisa foi aprovado pelo Comitê de Ética sob numero 014/97.

\section{RESULTADOS}

0 exame das respostas dadas, mostra que se obteve apenas 740 questionários com respostas válidas, entre os 767 preenchidos. Nos 27 questionários excluídos, não foi possível determinar a idade nem a indicações da residência.

As frequiências das características dos participantes e a caracterização dos diversos locais de nascimento e residência relevantes para a doença de Chagas, são apresentadas nas Tabelas 1 e 2. São também nelas exibidos os intervalos de confiança das estimativas percentuais.

As freqüiências de três grupos etários, definidos com base na história do controle constam da Tabela 3. Os grupos etários em que estão distribuídos os participantes obedecem, aproximadamente, as distintas fases do controle. 
TABELA 1

Distribuição de freqüências das características pessoais dos participantes do estudo.

\begin{tabular}{|c|c|c|c|c|}
\hline Características pessoais & Número & Percentagem & IC & $5 \%$ \\
\hline \multicolumn{5}{|l|}{ Sexo } \\
\hline feminino & 470 & 64,5 & 60,9 & 68,1 \\
\hline masculino & 259 & 35,5 & 32,0 & 39,1 \\
\hline \multicolumn{5}{|l|}{ Faixa etária } \\
\hline $07-25$ & 351 & 47,4 & 44,0 & 50,8 \\
\hline $26-50$ & 241 & 32,6 & 29,4 & 35,7 \\
\hline $51-85$ & 148 & 20,0 & 16,8 & 23,2 \\
\hline \multicolumn{5}{|c|}{ Parentesco com o responsável pelo domicílio } \\
\hline cônjuge & 160 & 22,6 & 19,7 & 25,6 \\
\hline filhos & 223 & 31,5 & 28,0 & 35,1 \\
\hline outros & 87 & 12,3 & 9,4 & 15,3 \\
\hline o próprio responsável & 237 & 33,5 & 30,2 & 36,8 \\
\hline \multicolumn{5}{|l|}{ Sabe ler e escrever } \\
\hline não & 176 & 24,5 & 20,9 & 28,2 \\
\hline $\operatorname{sim}$ & 541 & 75,5 & 71,8 & 79,1 \\
\hline \multicolumn{5}{|l|}{ Freqüenta escola atualmente } \\
\hline não & 293 & 51,5 & 47,1 & 55,9 \\
\hline $\operatorname{sim}$ & 276 & 48,5 & 44,1 & 52,9 \\
\hline \multicolumn{5}{|c|}{ Curso mais avançado já feito } \\
\hline médio & 109 & 20,0 & 16,3 & 23,6 \\
\hline primário & 429 & 78,6 & 74,8 & 82,3 \\
\hline superior & 8 & 1,5 & 0,5 & 2,5 \\
\hline
\end{tabular}

IC95\%: intervalo de confiança 95\%

Na Tabela 4 e Figura 1 são mostradas as frequiências das respostas dadas às questões relacionadas ao vetor, segundo o grupo de idade. A população com 51 e mais anos seria aquela nascida em período anterior às ações sistematizadas de controle; aquela entre os 16 e 50 anos conviveu com a transmissão endêmica da infecção chagásica e acompanhou o trabalho intensivo de controle vetorial; e, aquela entre 7 e 15 anos, corresponde à população que nasceu quando já controlada a transmissão domiciliar. A Figura 2 mostra o percentual de respostas relacionadas à conduta, diante do achado do vetor, segundo o grupo de idade.

A hipótese inicial era que o conhecimento existente estaria relacionado à idade dos participantes e à experiência vivida em relação à doença e sua transmissão pelo vetor. Quando se perguntou, você conbece o barbeiro? Os que responderam não e sim tinham média de idade de 14,1 e 36,1 anos respectivamente, diferença estatisticamente significante. Os que o conheciam de já tê-lo visto e os que o viram em sua própria casa tinham média de idade de 39,5 e 41,4 anos, respectivamente.

Considerando os mesmos grupos etários, arbitrados em função da cronologia do controle na área, na Tabela 5 são exibidas as freqüências dos tipos de respostas dadas pelos integrantes da amostra a questões relacionadas à doença de Chagas, seu mecanismo de transmissão, manifestações clínicas e prevenção. A Figura 3 exibe o percentual das respostas a uma das questões, dadas pelos participantes da amostra segundo o grupo de idade.
TABELA 2

Distribuição de frequiências das características dos locais de nascimento e residências atual e anteriores dos participantes do estudo.

\begin{tabular}{|c|c|c|c|c|}
\hline Características demográficas & Número & Percentagem & \multicolumn{2}{|c|}{ IC95\% } \\
\hline \multicolumn{5}{|l|}{ Município de residência } \\
\hline Buritinópolis & 259 & 35,0 & 29,8 & 40,3 \\
\hline Mambaí & 481 & 65,0 & 59,8 & 70,2 \\
\hline \multicolumn{5}{|l|}{ Zona de residência } \\
\hline rural & 251 & 33,9 & 28,7 & 39,1 \\
\hline urbana & 489 & 66,1 & 60,9 & 71,3 \\
\hline \multicolumn{5}{|l|}{ Município de nascimento } \\
\hline Buritinópolis & 72 & 9,9 & 7,3 & 12,5 \\
\hline Mambaí & 247 & 33,9 & 29,9 & 38,0 \\
\hline outros & 409 & 56,2 & 52,3 & 60,1 \\
\hline \multicolumn{5}{|l|}{ Zona de nascimento } \\
\hline rural & 344 & 55,5 & 51,1 & 59,8 \\
\hline urbana & 276 & 44,5 & 40,2 & 48,9 \\
\hline \multicolumn{5}{|c|}{ Tempo de residência no município atual } \\
\hline $00-09$ & 165 & 26,3 & 22,2 & 30,3 \\
\hline $10-25$ & 279 & 44,4 & 40,2 & 48,7 \\
\hline $26-96$ & 184 & 29,3 & 25,4 & 33,2 \\
\hline \multicolumn{5}{|l|}{ Zona de residência anterior } \\
\hline rural & 143 & 54,0 & 46,7 & 61,2 \\
\hline urbana & 122 & 46,0 & 38,8 & 53,3 \\
\hline \multicolumn{5}{|l|}{ Residência anterior (UF) } \\
\hline Bahia & 63 & 36,0 & 27,2 & 44,8 \\
\hline Distrito Federal & 23 & 13,1 & 6,6 & 19,7 \\
\hline Goiás & 59 & 33,7 & 25,7 & 41,7 \\
\hline Minas Gerais & 13 & 7,4 & 2,9 & 12,0 \\
\hline Mato Grosso do Sul & 1 & 0,6 & 0,6 & 1,7 \\
\hline Mato Grosso & 1 & 0,6 & 0,6 & 1,7 \\
\hline Pará & 2 & 1,1 & 0,5 & 2,8 \\
\hline Pernambuco & 2 & 1,1 & 0,5 & 2,8 \\
\hline Piauí & 1 & 0,6 & 0,6 & 1,7 \\
\hline Rio Grande do Norte & 1 & 0,6 & 0,6 & 1,7 \\
\hline São Paulo & 5 & 2,9 & 0,5 & 6,2 \\
\hline Tocantins & 4 & 2,3 & 0,4 & 5,0 \\
\hline
\end{tabular}

UF: unidade da federação, IC95\%: intervalo de confiança $95 \%$

TABELA 3

Distribuição de frequiências dos participantes do estudo segundo grupos de idade de interesse para a análise dos resultados.

\begin{tabular}{lcccr}
\hline Faixa etária & Número & Percentagem & \multicolumn{2}{c}{ IC95\% } \\
\hline $07-15$ & 212 & 28,6 & 25,1 & 32,2 \\
$16-50$ & 380 & 51,4 & 47,6 & 55,1 \\
$51-85$ & 148 & 20,0 & 16,8 & 23,2 \\
\hline
\end{tabular}

IC95\%: intervalo de confiança 95\%

Na Figura 4 observa-se a média de idade dos participantes que responderam sim ou não a uma questão particular sobre a doença de Chagas. E, finalmente, a Figura 5 mostra 0 conhecimento dos participantes de 7 a 15 anos, sobre o vetor e a doença de Chagas.

Os dados reunidos e o tratamento dado permitem a análise pretendida e com isso validam o questionário aplicado. 
TABELA 4

Frequiências das respostas às questões relacionadas ao inseto vetor dadas por integrantes de três grupos etários da amostra.

\begin{tabular}{|c|c|c|c|c|c|c|c|c|}
\hline \multirow{3}{*}{ Questões sobre o vetor } & \multicolumn{6}{|c|}{ Grupo etário } & \multirow{3}{*}{$\chi^{2^{*}}$} & \multirow{3}{*}{$\mathrm{P}$} \\
\hline & \multicolumn{2}{|c|}{$07-15$} & \multicolumn{2}{|c|}{$16-50$} & \multicolumn{2}{|c|}{$51-85$} & & \\
\hline & $\mathrm{n}^{0}$ & $\%$ & $\mathrm{n}^{0}$ & $\%$ & $\mathrm{n}^{\mathrm{o}}$ & $\%$ & & \\
\hline \multicolumn{9}{|l|}{ Você conhece o barbeiro? } \\
\hline não & 110 & 52,6 & 42 & 11,1 & 0 & 0,0 & 83,91 & $<0,01$ \\
\hline $\operatorname{sim}$ & 99 & 47,4 & 336 & 88,9 & 147 & 100,0 & & \\
\hline \multicolumn{9}{|l|}{ O conhece de onde? } \\
\hline cartaz/calendário & 21 & 22,3 & 49 & 14,3 & 5 & 3,4 & 6,41 & $<0,01$ \\
\hline informação do agente saúde & 4 & 4,3 & 6 & 1,8 & 0 & 0,0 & & \\
\hline informação no centro saúde & 3 & 3,2 & 4 & 1,2 & 0 & 0,0 & & \\
\hline informação escolar & 11 & 11,7 & 14 & 4,1 & 0 & 0,0 & & \\
\hline Já viu o inseto? & 55 & 58,5 & 269 & 78,7 & 141 & 96,6 & & \\
\hline \multicolumn{9}{|l|}{ Onde foi que o viu? } \\
\hline galinheiro & 3 & 5,5 & 14 & 5,2 & 6 & 4,3 & 0,87 & 0,536 \\
\hline mato & 3 & 5,5 & 15 & 5,6 & 7 & 5,0 & & \\
\hline outra casa & 11 & 20,0 & 49 & 18,2 & 19 & 13,6 & & \\
\hline outro local & 2 & 3,6 & 15 & 5,6 & 2 & 1,4 & & \\
\hline sua casa & 36 & 65,5 & 176 & 65,4 & 106 & 75,7 & & \\
\hline \multicolumn{9}{|l|}{ Outros locais onde viu o inseto } \\
\hline escola & 0 & 0,0 & 4 & 30,8 & 4 & - & 0,52 & 0,600 \\
\hline FUNASA-SUCAM & 1 & 50,0 & 3 & 23,1 & - & & & \\
\hline indeterminados & 1 & 50,0 & 6 & 46,2 & - & & & \\
\hline \multicolumn{9}{|c|}{ Sabe o que fazer quando encontrar o barbeiro? } \\
\hline avisar o agente de saúde & 35 & 31,0 & 268 & 74,9 & 111 & 75,5 & 16,78 & $<0,01$ \\
\hline avisar o centro de saúde & 4 & 3,5 & 16 & 4,5 & 3 & 2,0 & & \\
\hline matar o inseto & 47 & 41,6 & 61 & 17,0 & 28 & 19,1 & & \\
\hline não sabe o que fazer & 27 & 23,9 & 13 & 3,6 & 5 & 3,4 & & \\
\hline \multicolumn{9}{|l|}{ Qual sua importância? } \\
\hline porque incomoda a gente & 3 & 2,6 & 11 & 3,1 & 2 & 1,4 & 11,43 & $<0,01$ \\
\hline não sabe & 41 & 35,7 & 35 & 9,8 & 7 & 4,8 & & \\
\hline não tem importância & 3 & 2,6 & 5 & 1,4 & 1 & 0,7 & & \\
\hline porque transmite doença & 68 & 59,1 & 308 & 85,8 & 136 & 93,2 & & \\
\hline
\end{tabular}

$\chi^{2^{*}:}$ qui-quadrado ajustado ao padrão de variação intra e interfamílias incluídas no estudo.

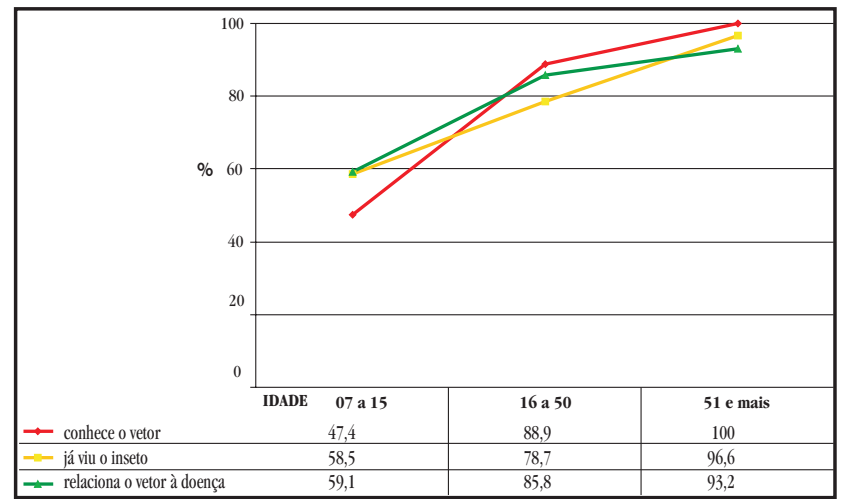

FIGURA 1

Proporção (\%) de respostas a questões relativas ao vetor dadas pelos participantes da amostra, segundo grupo de idade.

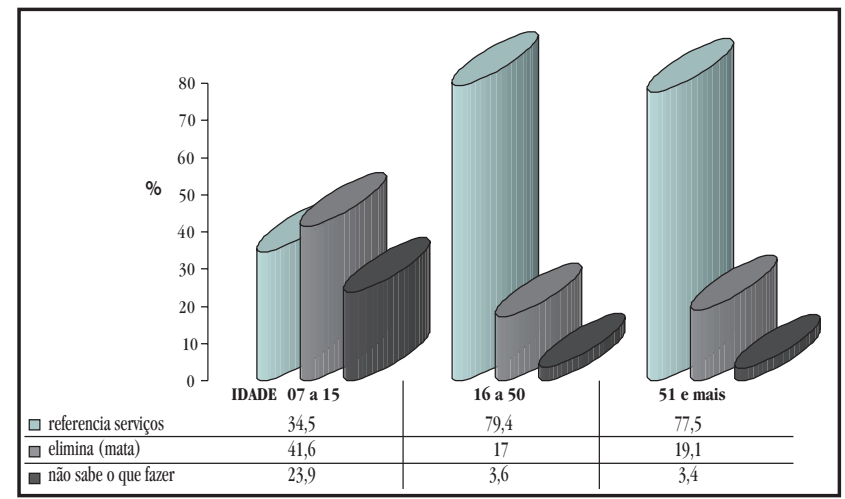

FIGURA 2

Proporção (\%) de respostas relativas à conduta a seguir diante do achado do vetor dadas pelos participantes da amostra, segundo grupo de idade. 
TABELA 5

Frequiências das respostas às questões relacionadas à doença de Chagas dadas por integrantes de três grupos etários da amostra.

\begin{tabular}{|c|c|c|c|c|c|c|c|c|}
\hline \multirow{3}{*}{ Questões a respeito da doença } & \multicolumn{6}{|c|}{ Grupo etário } & \multirow{3}{*}{$\chi^{2}$} & \multirow{3}{*}{$\mathrm{P}$} \\
\hline & \multicolumn{2}{|c|}{$07-15$} & \multicolumn{2}{|c|}{$16-50$} & \multicolumn{2}{|c|}{$51-85$} & & \\
\hline & $\mathrm{n}^{\underline{0}}$ & $\%$ & $\mathrm{n}^{\underline{0}}$ & $\%$ & $\mathrm{n}^{\underline{0}}$ & $\%$ & & \\
\hline \multicolumn{9}{|l|}{ Qual doença ele (o barbeiro) transmite? } \\
\hline doença de Chagas & 40 & 58,0 & 295 & 90,5 & 123 & 88,5 & 14,23 & $<0,01$ \\
\hline malária & 2 & 2,9 & 6 & 1,8 & 5 & 3,6 & & \\
\hline outra & 27 & 39,1 & 25 & 7,7 & 11 & 7,9 & & \\
\hline \multicolumn{9}{|c|}{ Você já ouviu falar da doença de Chagas? } \\
\hline não & 142 & 69,6 & 37 & 9,8 & 3 & 2,0 & 151,6 & $<0,01$ \\
\hline $\operatorname{sim}$ & 62 & 30,4 & 340 & 90,2 & 144 & 98,0 & & \\
\hline \multicolumn{9}{|c|}{ Quais órgãos são mais afetados pela doença? } \\
\hline coração & 37 & 56,9 & 282 & 86,8 & 111 & 83,5 & 5,89 & $<0,01$ \\
\hline intestino & 2 & 3,1 & 7 & 21,5 & 4 & 3,0 & & \\
\hline outros & 20 & 30,8 & 28 & 8,6 & 14 & 10,5 & & \\
\hline pulmão & 6 & 9,2 & 8 & 2,5 & 4 & 3,0 & & \\
\hline \multicolumn{9}{|l|}{ A doença de Chagas tem cura? } \\
\hline não & 22 & 23,7 & 160 & 45,6 & 65 & 44,8 & 4,27 & 0,002 \\
\hline não sabe & 30 & 32,3 & 86 & 24,5 & 45 & 31,0 & & \\
\hline $\operatorname{sim}$ & 41 & 44,1 & 105 & 29,9 & 35 & 24,1 & & \\
\hline \multicolumn{9}{|c|}{ Como a gente "pega" a doença de Chagas? } \\
\hline em contato com barbeiro & 44 & 80,0 & 286 & 95,7 & 120 & 97,6 & 5,27 & $<0,01$ \\
\hline em contato com doentes & 3 & 5,5 & 0 & 0,0 & 1 & 0,8 & & \\
\hline em contato com mosquitos & 6 & 10,9 & 9 & 3,0 & 2 & 1,6 & & \\
\hline não sabe & 2 & 3,6 & 4 & 1,3 & 0 & 0,0 & & \\
\hline \multicolumn{9}{|l|}{ Como evitar a doença de Chagas? } \\
\hline fervendo água para beber & 5 & 2,8 & 3 & 0,8 & 1 & 0,7 & 19,24 & $<0,01$ \\
\hline conservando e limpando a casa & 26 & 14,7 & 178 & 48,6 & 77 & 52,7 & & \\
\hline não sabe & 134 & 75,7 & 94 & 25,7 & 36 & 24,7 & & \\
\hline aplicando inseticida na casa & 9 & 5,1 & 83 & 22,7 & 31 & 21,2 & & \\
\hline vacinando as pessoas & 3 & 1,7 & 8 & 2,2 & 1 & 0,7 & & \\
\hline
\end{tabular}

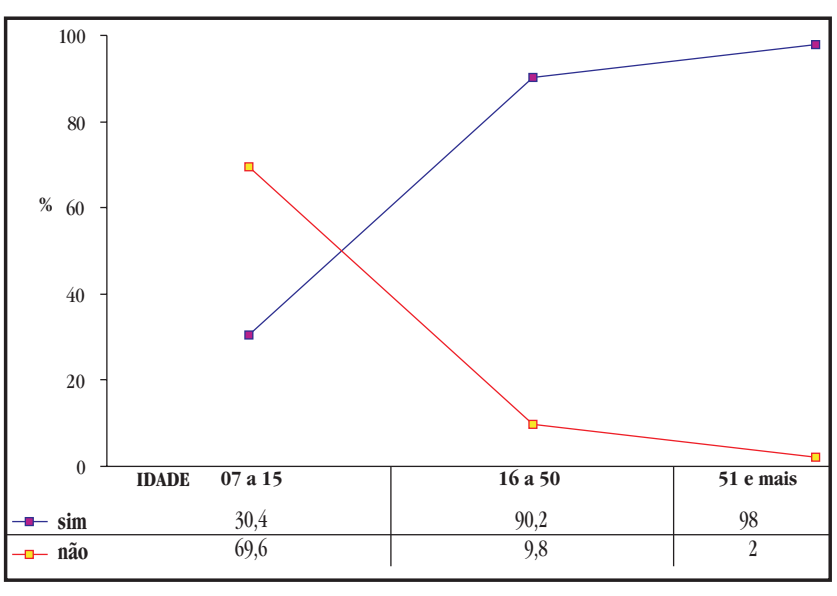

FIGURA 3

Proporção (\%) de respostas sobre o conhecimento ou não da doença de Chagas (Você já ouviu falar da doença de Chagas?) dadas pelos participantes da amostra, segundo grupo de idade.

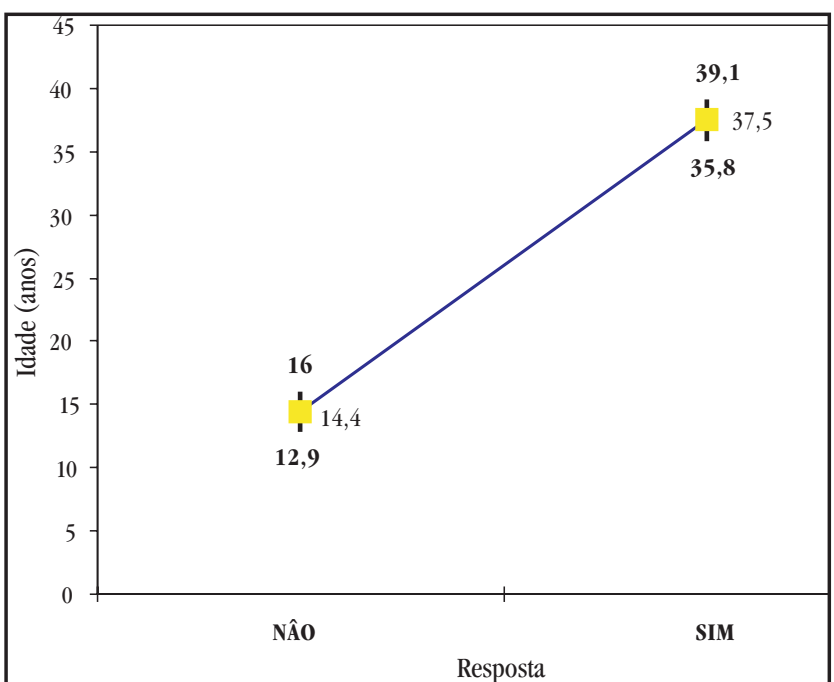

FIGURA 4

Idade média dos participantes da amostra que deram respostas sim e não à questão Você já ouviu falar da doença de Chagas? 


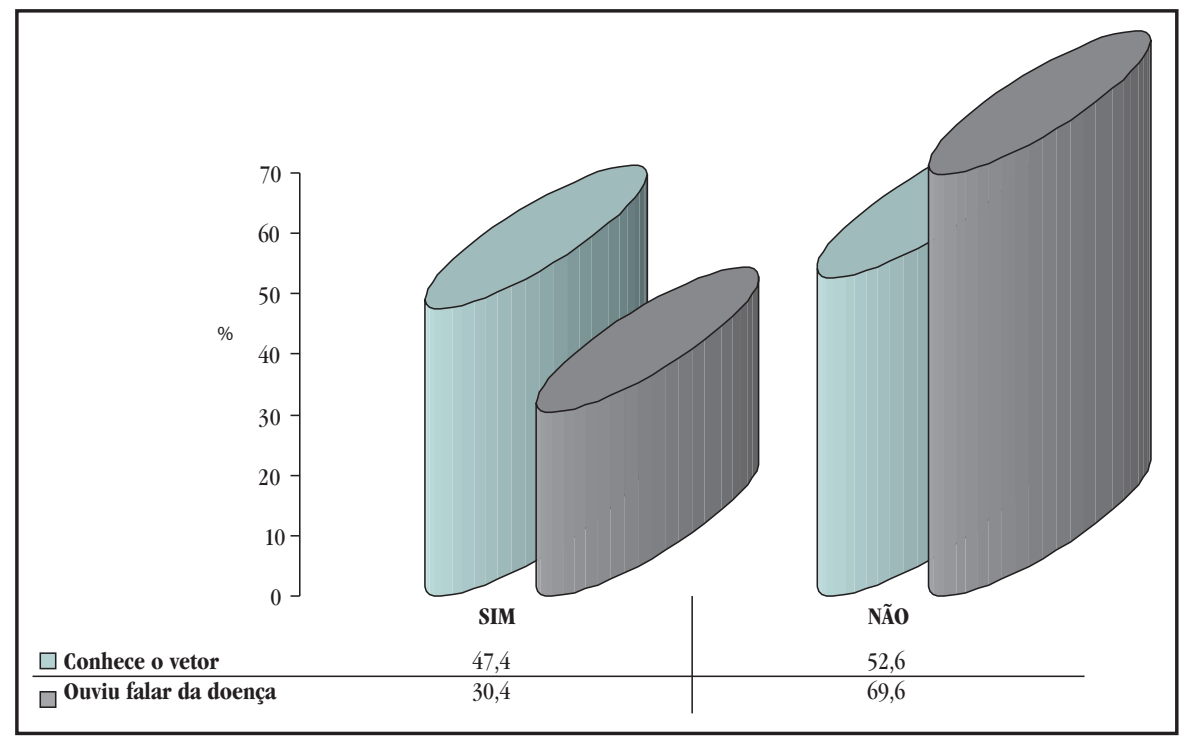

FIGURA 5

Proporção (\%) de participantes da amostra com idade de 7 a 15 que conhece e não conhece o vetor e que ouviu e não ouviu falar da doença de Chagas.

\section{DISCUSSÃO}

Uma consideração inicial é que o risco representado pela doença de Chagas foi reduzido, o que foi demonstrado de forma inquestionável nos trabalhos que compõem a avaliação das atividades na área ${ }^{1318}$. A possibilidade de restabelecimento da transmissão vetorial no ambiente domiciliar em Mambaí e Buritinopolis, é remota. Poderia se dar pela reintrodução de Triatoma infestans desde áreas ainda infestadas ou pela colonização intradomiciliar de espécies nativas (Triatoma sordida e Rhodnius neglectus, seriam eventuais candidatas). Em ambos os casos isso parece improvável, considerando que: $1^{\circ}$ ) já há algum tempo não se tem verificado a presença de Triatoma infestans em áreas geograficamente próximas; $\mathrm{e}, 2^{\circ}$ ) as espécies candidatas à substituição de Triatoma infestans não reúnem os atributos necessários para atuarem como vetores capazes de sustentar a transmissão endêmica do Trypanosoma cruzi ao homem; especialmente pela sua marcada ornitofilia, do que decorrem baixas taxas de infecção natural e pouca tendência à constituição de colônias no interior dos domicílios. Muito embora há registros, de persistência ou recrudecencia de focos domiciliares em regiões do Oeste de Minas Gerais que estavam sob vigilância epidemiológica ${ }^{20}$.

Obviamente, a visibilidade da doença, que mais explicitamente se relaciona à presença do vetor nas habitações, e que é já tão pouco evidente, não é mais a mesma na área objeto de estudo. Não se poderia pretender que com a ausência de transmissão e, com o desaparecimento do Triatoma infestans, a população se mantivesse informada e interessada no tema.

É necessário, entretanto, levar em conta as características dos participantes do estudo na avaliação das respostas dadas às questões. Os resultados das Tabelas $\mathbf{1 , 2} \mathbf{2} \mathbf{3}$, indicam que a maioria dos respondentes era o próprio responsável pelo domicílio, pertencente ao gênero feminino e residente nas zonas urbanas de Mambaí e Buritinópolis. A maioria deles encontrava-se entre 16 e 50 anos de idade, possuía instrução primária, havia nascido na zona rural e residia na área há pelo menos 10 anos. Cerca de $43,8 \%$ nasceram nos municípios onde residiam e 56,2\% em outros municípios. A maioria também tinha a última residência, antes da atual, na zona rural. Dentre os que migraram para a região, mais de $60 \%$ vieram da Bahia ou de outros municípios de Goiás. As características mencionadas indicam que predominam entre os participantes do estudo indivíduos familiarizados com a doença de Chagas e com a presença dos agentes do controle da doença na região. Em virtude disso, é esperado que as respostas dadas a respeito da doença e seu transmissor, possam refletir a experiência dos entrevistados com vários aspectos da endemia e seu controle.

Tomando os dados constantes da Tabela 4 e Figura 1, relativos ao vetor, e considerando a distribuição das respostas por grupo de idade verifica-se que $100 \%$ da população maior de 50 anos afirma conhecer o inseto, dos quais $96,6 \%$ já o teriam visto; enquanto aproximadamente $50 \%$ dos indivíduos entre 7 e 15 anos dizem conhecer o inseto, sendo que grande parte deles (34\%) apenas por informação, na escola ou através de cartazes.

É evidente que o conhecimento do vetor é variável dependente da idade, assim como a forma de conhecimento ou informação difere com a idade. Ainda assim, da população nascida depois do controle da transmissão, e que informa conhecer o vetor, uma proporção importante afirma conhecê-lo de sua própria casa $(65,5 \%)$. Além disso, interessa notar que essa é variável pouco influenciada pela idade. Isso é compreensível pelo fato de que 0 Triatoma sordida é espécie autóctone da região, e que a invasão esporádica dos insetos é acontecimento esperado, sem que isso represente maior risco. Por outro lado, considerando-se apenas os indivíduos que dizem haver em algum momento observado 0 vetor em sua casa a idade média é de 41,4 anos. A princípio julgava-se que houvesse a percepção de que 0 vetor se encontra mais frequentemente em galinheiros, em função de sua associação $\mathrm{a}$ aves, e isso não se confirmou (Tabela 4). 
Em relação ao nexo vetor-transmissão-doença, nota-se que outra vez os grupos de maior idade o estabelece com mais frequiência $(93,2 \%$ entre aqueles participantes com mais de 50 anos e de $85,8 \%$ dos com idade entre os 16 e 50 anos). Note-se ainda que uma proporção significativa $(35,7 \%)$ de indivíduos menores de 15 anos diz não saber que importância tem o vetor.

Com respeito a providencias a tomar diante do achado do vetor observa-se a desinformação do grupo de menor idade. Deve-se destacar o fato de que aproximadamente $25 \%$ da população entre $7 \mathrm{e}$ 15 anos não sabe o que fazer, enquanto cerca de $80 \%$ da população adulta, com idade superior aos 15 anos, diz notificar ou referenciar informação aos serviços de saúde (Tabela 4 e Figura 2).

Com base nesses resultados, pode-se concluir que a população jovem está pouco informada sobre o vetor e sua relação com a doença de Chagas, bem como em sua maioria não tem referências claras sobre como agir diante de seu achado. Isso seria atribuível ao fato de que não vivenciaram a doença como problema, combatido de forma intensiva pelos serviços. Além disso, deve-se considerar que a vigilância instituída na área tomou o responsável pelo núcleo familiar como referente, diversamente do modelo de vigilância, difundido em outras regiões, baseado na participação da comunidade escolar.

Quando o tema tratado foi a própria doença de Chagas, suas manifestações, prevenção e possibilidade de tratamento, as diferenças entre os grupos etários foram igualmente notáveis. Apenas 2\% do grupo acima dos 50 anos não ouviu falar da doença de Chagas, enquanto a proporção de indivíduos de 7 a 15 que deu a mesma resposta alcançou a cifra de 69,6\% (Tabela 5 e Figura 3), e tinha idade média de 14,4 anos (Figura 4).

Um dado inesperado foi a desproporção entre os jovens que informaram conhecer o vetor e aqueles que disseram conhecer a doença (Figura 5). Essa aparente inconsistência seria explicada pelo fato de não fazerem claramente a conexão do vetor com a doença.

Como conclusão, e fazendo uma projeção para o futuro da vigilância entomológica da doença de Chagas em áreas com transmissão interrompida, deve-se ter claro que não se pode pretender que o envolvimento da população seja o mesmo daquele em áreas em que a doença, e especialmente o vetor, estão presentes. Uma evidencia disso, foi o diferente entendimento da população em função da idade. Haverá sempre a tendência de que a população nascida após o controle não participe da vigilância de uma forma mais comprometida.

Essas considerações remetem à apreciação de que a vigilância entomológica, em áreas com transmissão interrompida, com baixo risco de re-introdução do vetor primário e, com poucas chances de colonização domiciliar por espécies nativas, seja basicamente sustentada por pesquisa domiciliar direta por pessoal institucional como ocorreu em parte no Estado de São Paulo entre 1984 e $1989^{14}$. A pesquisa periódica, pode ser feita em parte por amostragem dirigida a localidades de maior risco e em parte aleatória ${ }^{19}$. A identificação das localidades de maior risco se faria a partir de sua vulnerabilidade, em função do tipo de habitação, mobilidade das populações e antecedentes históricos. Os dados disponíveis indicam que, de modo geral, o risco é muito baixo. Com isso a periodicidade da busca poderia ser bienal ou trienal, e o principal objeto de vigilância, deve ser a detecção precoce de qualquer indicio de colonização domiciliar por Triatoma sordida.

Ainda que a oportunidade de detecção do vetor seja maior com a vigilância baseada na notificação pela comunidade, a pouca motivação que progressivamente tende a população em uma situação como a vigente na área de estudo, inviabiliza a possibilidade de uma vigilância fundada exclusivamente na participação comunitária. E pelo que recomendam os resultados colhidos, deve ser posta maior atenção na participação das escolas.

Outra apreciação diz respeito ao que é preconizado em termos de controle químico de espécies nativas com limitada capacidade vetorial, caso do Triatoma sordida e de outras espécies autóctones da área ${ }^{1621}$. Apenas se indica o tratamento domiciliar com inseticidas quando se comprova a existência de colônias intradomiciliares. Em função disso, a vigilância entomológica através da notificação pela população poderá criar uma extraordinária, demanda. A presença esporádica de exemplares adultos desses triatomíneos na habitação, ou colonizando o peridomicílio, é previsível, em se tratando de espécies nativas. Tomando-se com rigor o que seria recomendado, não exige a princípio nenhuma intervenção, a menos que se comprove a existência de grandes colônias peridomiciliares que representem pressão sobre o domicílio.

\section{REFERÊNCIAS}

1. Bizerra JF, Gazzana MR, Costa CH, Mello DA, Marsden PD. A survey of what people know about Chagas' disease. World Health Forum 2: 394-397, 1981.

2. Busvine JR, Barnes S. Observations on mortality among insects exposed to dry insecticidal films. Bulletin of Entomological Research 38: 80-81, 1947.

3. Castro CN. Influência da parasitemia no quadro clínico da doença de Chagas. Revista de Patologia Tropical 9: 73-136, 1980.

4. Dias E, Pellegrino J. Alguns ensaios com o "Gamexanne" no combate aos transmissores da doença de Chagas. Brazil Médico 62: 185-190, 1948.

5. Garcia-Zapata MT, Marsden P. Enfermedad de Chagas: control y vigilância com insecticidas y participación comunitaria en Mambaí, Goiás, Brasil. Boletín De La Oficina Sanitaria Panamericana 116: 97-110, 1994.

6. Marsden PD. The control of Chagas' Disease in Mambaí, Brazil: the initial phases. Infection Control 2: 466-470, 1981.

7. Marsden PD, Garcia-Zapata MT, Castillo EAS, Prata A, Macêdo V. Los 13 primeros años del control de la enfermedad de Chagas en Mambaí, Goiás, Brasil, 19801992. Boletín De La Oficina Sanitaria Panamericana 116: 111-117, 1994.

8. Marsden PD, Penna R. A 'vigilance unit' for households subject to triatomine control. Transactions of the Royal Society of Tropical Medicine and Hygiene 76: 790-792, 1982.

9. Marsden PD, Virgens D, Castro CN, Brasil IP, Ferreira R, Silveira AC, Matos CA, Macedo V, Prata A. The Control of Chagas' Disease Transmission in Mambaí, Goiás-Brazil (1980-1981). Revista da Sociedade Brasileira de Medicina Tropical 16: 189-195, 1983.

10. Marsden PD, Virgens D, Magalhães I, Tavares-Neto J, Pereira R, Costa CH, Castro CN, Macêdo V, Prata A. Ecologia doméstica do Triatoma infestans em Mambaí, GoiásBrasil. Revista do Instituto de Medicina Tropical de São Paulo 24: 364-373, 1982.

11. Moreno EC, Baracho L. Vigilância epidemiológica no Programa de Controle da Doença de Chagas em Minas Gerais, Brasil (1984-1998). Cadernos de Saúde Pública 16 (supl 2):113-116, 2000. 
12. Organización Panamericana de la Salud. Taller del Cono Sur sobre enfermedad de Chagas. Conceptualizacion de la Vigilancia Epidemiologica. Buenos Aires, Argentina, septiembre 2003. Montevideo: OPS. (OPS/DPC/CD/290/04), 2004.

13. Peñaranda-Carrillo R, Moreira EF, Silveira AC, Leite J, Vinhaes MC, Castro C, Macedo V. Avaliação do impacto das ações de controle vetorial da doença de Chagas através de inquérito sorológico em Mambaí/Buritinópolis, Goiás. Revista da Sociedade Brasileira de Medicina Tropical 35: 331-338, 2002.

14. Rocha e Silva EO, Wanderley DMV, Rodrigues VLCC. Triatoma infestans: importância, controle e eliminação da espécie no Estado de São Paulo, Brasil. Revista da Sociedade Brasileira de Medicina Tropical 31: 73-88, 1998.

15. Romaña C, Abalos JW. Acción del "Gamexanne" sobre los triatomídeos. Control domiciliario. Anales del Instituto de Medicina Regional, Tucuman 2: 95-106, 1948.

16. Silveira AC. Profilaxia. In: Brener Z, Andrade ZA, Barral Neto M (orgs) Trypanosoma cruzi e Doença de Chagas. $2^{\text {a }}$ edição. Guanabara Koogan, Rio de Janeiro, p. 75-87, 1999.

17. Silveira AC. Modelos alternativos de vigilância e controle da doença de Chagas para fases avançadas dos Programas. Grupo de Trabajo OPS en Enfermedad de
Chagas. Montevideo, Uruguay, noviembre de 2001. Available from URL: http:// www.paho.org/spanish/ad/dpc/cd/consulta-2.pdf, 2001.

18. Silveira AC, Peñaranda-Carrillo R, Lorosa ES, Leite J, Vinhaes MC, Castro CN, Prata A, Macedo V. Evaluation of the impact of chemical control measures and entomological surveillance on Chagas' disease in the counties of Mambaí and Buritinópolis, Goiás State, Brazil. Revista da Sociedade Brasileira de Medicina Tropical 34: 549-557, 2001.

19. Silveira AC, Sanches O. Guía para Muestreo en Actividades de Vigilancia y Control Vectorial de la Enfermedad de Chagas. Organización Panamericana de la Salud, (OPS/DPC/CD/276/03), 2003

20. Villela MM, Souza JMB, Melo VP, Dias JCP. Vigilância epidemiológica da doença de Chagas em programa descentralizado: avaliação de conhecimentos e práticas de agentes municipais em região endêmica de Minas Gerais, Brasil. Cadernos de Saúde Pública 23: 2428-2438, 2007.

21. World Health Organization. Control of Chagas Disease. (WHO Technical Report Series 905), 2002. 